\title{
A Chimera Analysis of Prestin Knock-Out Mice
}

\author{
Mary Ann Cheatham, ${ }^{1}$ Sharon Low-Zeddies, ${ }^{3}$ Khurram Naik, ${ }^{1}$ Roxanne Edge, ${ }^{1}$ Jing Zheng, ${ }^{1}$ Charles T. Anderson, ${ }^{1}$ \\ and Peter Dallos ${ }^{1,2}$ \\ Departments of ${ }^{1}$ Communication Sciences and Disorders, The Hugh Knowles Center and ${ }^{2}$ Neurobiology and Physiology, Northwestern University, \\ Evanston, Illinois 60208, and ${ }^{3}$ MusWorks, Falls Church, Virginia 22042
}

\begin{abstract}
A chimera is a genetic composite containing a unique mix of cells derived from more than one zygote. This mouse model allows one to learn how cells of contrasting genotype functionally interact in vivo. Here, we investigate the effect that different proportions of prestin-containing outer hair cells $(\mathrm{OHC})$ have on cochlear amplification. To address this issue, we developed a prestin chimeric mouse in which both ROSA26 wild-type (WT) and prestin knock-out (KO) genotypes are present in a single cochlea. The WT ROSA26 mice express a cell marker, allowing one to identify cells originating from the WT genome. Examination of cochlear tissue indicated that prestin chimeric mice demonstrate a mosaic in which mutant and normal OHCs interleave along the cochlear partition, similar to all other chimeric mouse models. The anatomical distribution of prestin-containing OHCs was compared with physiological data including thresholds and tuning curves for the compound action potential (CAP) recorded in anesthetized mice. Analysis of these measures did not reveal mixed phenotypes in which the distribution of prestin-containing OHCs impacted sensitivity and frequency selectivity to different degrees. However, by reducing the number of prestin-containing OHCs, phenotypes intermediate between WT and KO response patterns were obtained. Accordingly, we demonstrate a proportional reduction in sensitivity and in the tip length of CAP tuning curves as the number of OHCs derived from the KO genome increases; i.e., genotype ratio and phenotype are closely related.
\end{abstract}

\section{Introduction}

The sensitivity and frequency selectivity of the auditory system is established in the cochlea and specifically at the mechanical level, which is apparently controlled by the outer hair cells (OHC). This knowledge is based on results showing that removal of OHCs results in threshold shift and loss of tuning (Ryan and Dallos, 1975; Dallos and Harris, 1978). This phenotype is also observed in mice lacking the prestin gene (Liberman et al., 2002; Cheatham et al., 2004) whose protein product is the OHC motor protein (Zheng et al., 2000), which is normally localized to the OHC's lateral membrane (Zheng et al., 2003). To further study cochlear amplification, the process underlying sensitivity and frequency selectivity has been characterized in various prestin mouse models. To date this includes the prestin knock-out (KO) mouse, as well as a knockin (KI) with mutated prestin that successfully targets the plasma membrane. All reports indicate that prestin is required for normal cochlear function. When prestin is removed (Liberman et al., 2002; Cheatham et al., 2004) or when it is not functioning properly (Dallos et al., 2008), there is a substantial loss in cochlear sensitivity and an absence of tuning, two of the hallmarks of cochlear feedback amplification. Unfortunately, none of the currently available mouse models is adequate to fully

Received April 3, 2009; revised Aug. 12, 2009; accepted Aug. 14, 2009.

This work was supported by National Institute on Deafness and Other Communication Disorders Grant DC00089 (to P.D.), National Institute of Mental Health Grant 5R44MH066670 (to S.L.Z.), and The Knowles Leadership Fund (to M.A.C.). We thank Lindsey Tengerstrom for help constructing cochleograms.

Correspondence should be addressed to Mary Ann Cheatham, 2-240 Frances Searle Building, 2240 Campus Drive, Northwestern University, Evanston IL 60208-3550. E-mail: m-cheatham@northwestern.edu. DOI:10.1523/JNEUROSCI.1651-09.2009

Copyright $\odot 2009$ Society for Neuroscience ～0270-6474/09/2912000-09\$15.00/0 address the question of the functional significance of OHC electromotility because $\mathrm{OHCs}$ in $\mathrm{KO}$ mice are much shorter and less stiff than in wild-type controls and because both $\mathrm{KI}$ and $\mathrm{KO}$ models suffer $\mathrm{OHC}$ death.

In this report, we describe a chimera analysis of prestin function. A chimera is a genetic composite. It contains a unique mix of cells derived from more than one zygote. Although first reported in the early 1960s (Tarkowski, 1961; Mintz, 1962), current interest relates to the availability of transgenic cell-marker strains (Friedrich and Soriano, 1991; Zambrowicz et al., 1997) and the profusion of new mouse mutants (Brown et al., 2008). This approach is important because it allows one to learn how cells of contrasting genotype functionally interact in vivo in a single cochlea. Because of differences in the specific contribution of each genome to a given tissue, the number of cells originating from either genome varies from animal to animal. As a result, each chimera is a unique individual where the distribution of the two cell populations can vary between $0 \%$ and $100 \%$ (Mullen and Whitten, 1971; Falconer and Avery, 1978). It is, therefore, possible to document the effect of differing numbers of prestin-containing OHCs on cochlear amplification and to determine dose relationships. To obtain this information, we created prestin chimeric mice by combining ROSA26 wildtype (WT) embryos with prestin $\mathrm{KO}$ embryos, allowing us to study prestin's contribution to cochlear amplification at the cellular level. Because ROSA26 mice contain a cell marker, it is possible to document the distribution of normal and mutant $\mathrm{OHCs}$ in a single cochlea and to correlate this distribution with cochlear physiology. 


\section{Chimera Construction}

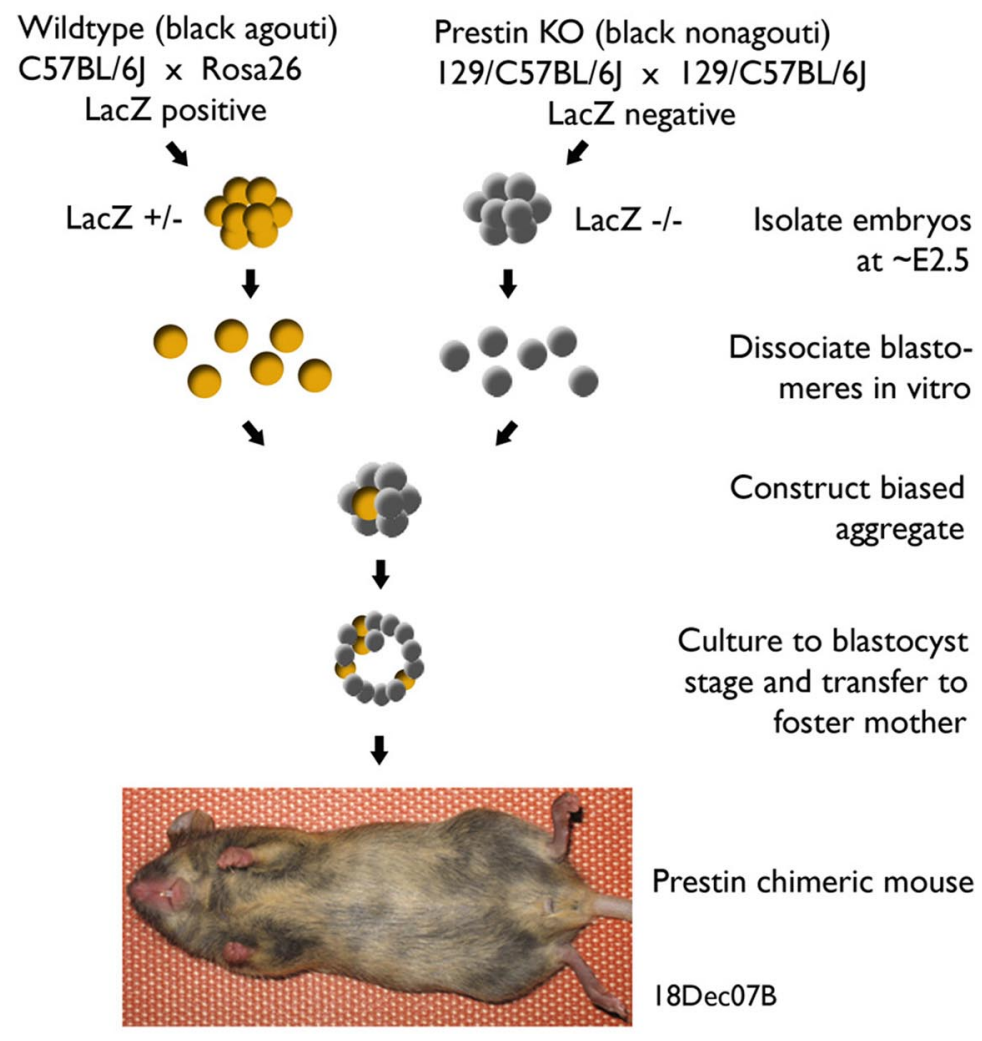

Figure 1. Construction of prestin chimeras. Parental strains with contrasting coat color were used to form two embryo groups differing in the expression of prestin and lacZ genes. After fusion of contrasting embryos, a chimeric blastocyst is transferred to a pseudopregnant female. Prestin chimeric mice are easily distinguished in any given litter by their variegated coat color.

wire ball electrode in anesthetized mice ( 80 $\mathrm{mg} / \mathrm{kg}$ sodium pentobarbital, i.p.) between postnatal days 27 and 44. Compound action potential (CAP) thresholds were obtained for a $10 \mu \mathrm{V} \mathrm{N} 1 / \mathrm{P} 1$ criterion voltage. CAP tuning curves were acquired at $12 \mathrm{kHz}$ using the simultaneous tone-on-tone masking technique (Dallos and Cheatham, 1976). For each individual tuning curve, the $12 \mathrm{kHz}$ probe tone was adjusted in level to produce a $25 \mu \mathrm{V} \mathrm{N} 1 / \mathrm{P} 1$ when presented in isolation. Masker level was then adjusted to produce a $3 \mathrm{~dB}$ decrease in this $12 \mathrm{kHz}$ response. A full description of these methods appears previously (Cheatham et al., 2004; Gao et al., 2007).

Histological processing. After death with an overdose of Euthasol $(200 \mathrm{mg} / \mathrm{kg})$, mice were cardiac perfused first with heparinized PBS and then with $4 \%$ paraformaldehyde. Cochleae were then harvested and postfixed for $\sim 2 \mathrm{~h}$ at room temperature. Because the number and size of the $\beta$-galactosidase positive inclusions can be quite variable (Friedrich et al., 1993), we used our affinity purified, C-terminal prestin antibody (anti-prestin, 1:2000) (Matsuda et al., 2004) in combination with a secondary antibody, donkey anti-rabbit IgG-HRP (horseradish peroxidase, 1:200) (Jackson ImmunoResearch Laboratories) and subsequent reaction with diaminobenzidine (DAB Substrate Kit for Peroxidase, Vector Laboratories). Cochleae were stained, dehydrated, and plasticized at $60^{\circ} \mathrm{C}$ overnight using an Araldite 502/PolyBed 812 kit (Polysciences). Excess resin and cochlear bone were removed before separating segments of the cochlear duct from the modiolus (Bohne and Harding, 1997) and mounting them on glass slides for observation using bright field, light

\section{Materials and Methods}

Generation of chimeras and controls. Separate breeding colonies were established for prestin $\mathrm{KO}$ and ROSA26 WT mice. Prestin mutant mice were obtained from the Mouse Mutant Regional Resource Center (University of North Carolina, Chapel Hill, NC) and displayed a KO phenotype (Anderson et al., unpublished observations). The ROSA26 mice are essentially C57BL6 while the prestin KOs are on a mixed 129/BL6 genetic background.

Because chimeras cannot be produced by breeding alone, the procedure used to construct a prestin chimera is shown in Figure 1. The prestin $\mathrm{KO}$ was black nonagouti or for simplicity black in color. The other parental strain was a ROSA26 heterozygote (het); i.e., a ROSA26 homozygote was originally crossed with a C57BL6 mouse. This mouse was WT at the prestin locus and black agouti or brown in coat color. It carries the cell marker, $\beta$-galactosidase, which is an enzyme produced by the lac $Z$ transgene. These 2 parental strains were used to form embryo groups that differ in the expression of prestin and the cell marker. In other words, the WT mouse was brown and expressed both prestin and lacZ genes; the KO was black and negative for these two genes. Embryos were harvested from each group at E2.5 when cells are totipotent (Kelly, 1977). Eight-cell blastomeres were aggregated (Hogan et al., 1994) and cultured to the blastocyst stage. Composite or chimeric blastocysts were then transferred into a foster mother. In any given litter, chimeric pups were identified by their variegated or mottled coat color. Although nonchimeric mice served as controls, we also developed an additional control group by mating a homozygous ROSA26 male with a prestin KO female. These controls, as well as all of the chimeric mice, were hemizygous for the lac $Z$ transgene.

Measurement of CAP thresholds and tuning curves. All procedures were approved by Northwestern's Institutional Animal Care and Use Committee, as well as by The National Institutes of Health. Gross cochlear potentials were acquired at the round window membrane using a silver microscopy. Because whole-mount tissue is thick, a single plane of focus did not suffice over the entire length of a given segment. Therefore, multiple images were captured, each at a different focal plane. These pictures were then combined using MicroSuite FIVE (Olympus Soft Imaging Solutions) and its Extended Focal Imaging (EFI) module to create a single in-focus image. Adjacent fields of view were subsequently stitched together from base to apex using DoubleTake (Echo One). The final image of all concatenated pictures showed each row of OHCs in focus. After calculating cochlear length and dividing the tissue into 7\% segments, cochleograms were constructed by plotting the average percentage of OHCs lacking prestin in each 7\% segment along the basilar membrane. In mice that had very few OHCs containing prestin, there were usually some missing cells in the base of the cochlea similar to prestin $\mathrm{KO}$ mice (Wu et al., 2004). In this case, the percentage of OHCs lacking prestin included the unstained cells, as well as the number of missing OHCs. Because segmentation started in the base, the first segment plotted at $93 \%$ extended from the very end of the basilar membrane to a location $7 \%$ of the distance from the base of the cochlea. The decision to plot our results by aligning the data in each bin to the lowest margin rather than to its center was made to be consistent with the original data published for prestin KO mice (Wu et al., 2004). Five-micron radial sections were also cut from re-embedded short cochlear segments and viewed using bright-field, light microscopy.

The procedures for X-Gal histochemistry were similar to those used for the clock chimera (Low-Zeddies and Takahashi, 2001). After fixation, apical cochlear coils were dissected and stained at $37^{\circ} \mathrm{C}$ overnight with a solution containing $1 \mathrm{mg} / \mathrm{ml} \mathrm{X-Gal} \mathrm{(5-bromo-4-chloro-3-indolyl-} \beta$-Dgalactopyranoside (Sigma-Aldrich) dissolved in dimethyl formamide), 5 $\mathrm{mm} \mathrm{K}_{3} \mathrm{Fe}(\mathrm{CN})_{6}$ and $5 \mathrm{~mm} \mathrm{~K}_{4} \mathrm{Fe}(\mathrm{CN})_{6}$ in wash buffer. After rinsing, the tissue was mounted with Fluoromount-G (Southern Biotech) on glass slides and viewed as a whole mount with light microscopy. 


\section{Results}

\section{Data from control mice}

Before beginning this series of experiments, we determined that the parental strain carrying the cell marker had normal cochlear sensitivity. This result indicates that the lacZ gene provides a developmentally neutral cell marker and that it does not influence the expression or the function of the prestin gene. As an additional control, we also mated mice from the two parental strains. The offspring are essentially prestin heterozygotes where the WT mouse is ROSA26 positive. Assuming that strain background is not a confounding factor, these ROSA26 WT/prestin $\mathrm{KO}$ controls would be expected to demonstrate WT-like sensitivity and tuning (Cheatham et al., 2005). Data in Figure 2 indicate that this is the case. CAP thresholds on the left and CAP tuning curves on the right are both similar to the average WT/het physiology. This result is consistent with our previously published results on prestin heterozygotes where it was shown that near-normal levels of prestin protein are expressed in mice having only one copy of the prestin gene (Cheatham et al., 2005). Results in Figure 2 also confirm that there is no negative outcome due to lac Z expression in cochlear hair cells or to strain background effects.

These ROSA26/prestin controls, as well as all of the chimeras, are heterozygous for the cell marker. In other words, they contain $\beta$-galactosidase, an enzyme produced by the lac $Z$ gene, which turns its substrate, $\mathrm{X}$-Gal, blue. In Figure 3, the whole mount on the left displays the apical turn of a ROSA26-positive cochlea. All cells display the blue reaction product, while the negative control on the right shows no stain.

\section{Data from chimeric mice}

Of the 54 mice available, 7 were brown indicating WT, 31 were black indicating $\mathrm{KO}$, and 16 mice were mottled indicating contributions from both parental strains. It is of interest that $69 \%$ of the mice were male, which compares with clock chimeras where $72 \%$ were also male (Low-Zeddies and Takahashi, 2001). Early experiments (Tarkowski, 1961; Mullen and Whitten, 1971) revealed that $\sim 50 \%$ of experimental embryos are male/female, $\sim 25 \%$ are male, and $\sim 25 \%$ are female. Because of the importance of the $\mathrm{Y}$ chromosome in determining sex, most of the sex chimeras (XX$\mathrm{XY}$ ) are phenotypically male. Hence, they should theoretically outnumber females by $3: 1$. Portraits were obtained on 46 of the 54 mice and ordered from black to brown, as shown in supplemental materials, available at www.jneurosci.org.

Anatomical data were obtained on 27 ears collected from 16 mice. The average basilar membrane length was $6.02 \pm 0.18 \mathrm{~mm}$ (mean \pm one SD) with each $7 \%$ segment covering $0.419 \pm 0.004$ $\mathrm{mm}$. The distribution of prestin-containing outer hair cells is with a prestin $\mathrm{KO}$ mouse.

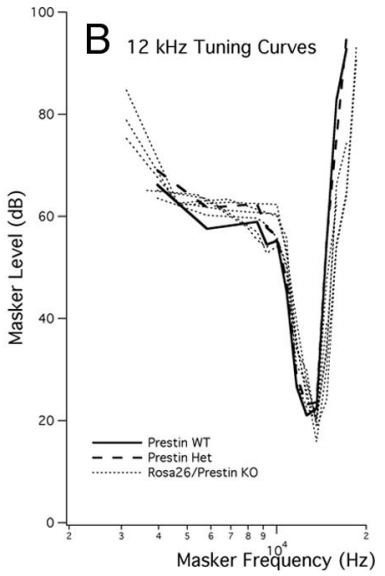

Figure 2. Peripheral auditory physiology is normal in ROSA26/prestin heterozygous mice. $A, B, 0$ ffspring obtained by mating a ROSA26 homozygote with a prestin KO mouse show normal CAP thresholds in $\boldsymbol{A}$ and CAP tuning curves in $\boldsymbol{B}$. WT data are plotted with solid lines, prestin heterozygotes with dashed lines, and ROSA26/prestin heterozygotes with dotted lines.

igure 3. LacZ staining in the organ of Corti. An apical coil is stained with the chromogenic substrate X-Gal in a ROSA26-positive mouse on the left and a negative control shown on the right at $25 \times$. These mice were obtained by breeding a ROSA26 homozygote

shown in Figure $4 A$. These images were obtained from a mottled mouse with $\sim 23 \%$ of OHCs immuno-positive for prestin. To evaluate the distribution of prestin-containing cells, a segment located $2.8-4.3 \mathrm{~mm}$ from the base is displayed. The enlarged region indicates that there is no propensity for cells of similar genotype to group together; i.e., there were no large patches of prestin-containing OHCs alternating with $\mathrm{OHCs}$ lacking prestin. This observation is consistent with reports that early aggregation chimeras produce mosaics in all tissue examined to date (Dewey et al., 1976; Oster-Granite and Gearhart, 1981; Goldowitz, 1987, Wang et al., 2006; Du et al., 2007).

We also obtained radial sections to better evaluate the architecture of the organ of Corti when stained and unstained cells occurred at the same location, as shown in Figure $4 \mathrm{~B}$. While OHCs lacking prestin are shorter than WT (Liberman et al., 2002; Cheatham et al., 2007), Deiters' cells increase in length, preventing gross distortions in the anatomical relationships between cells 


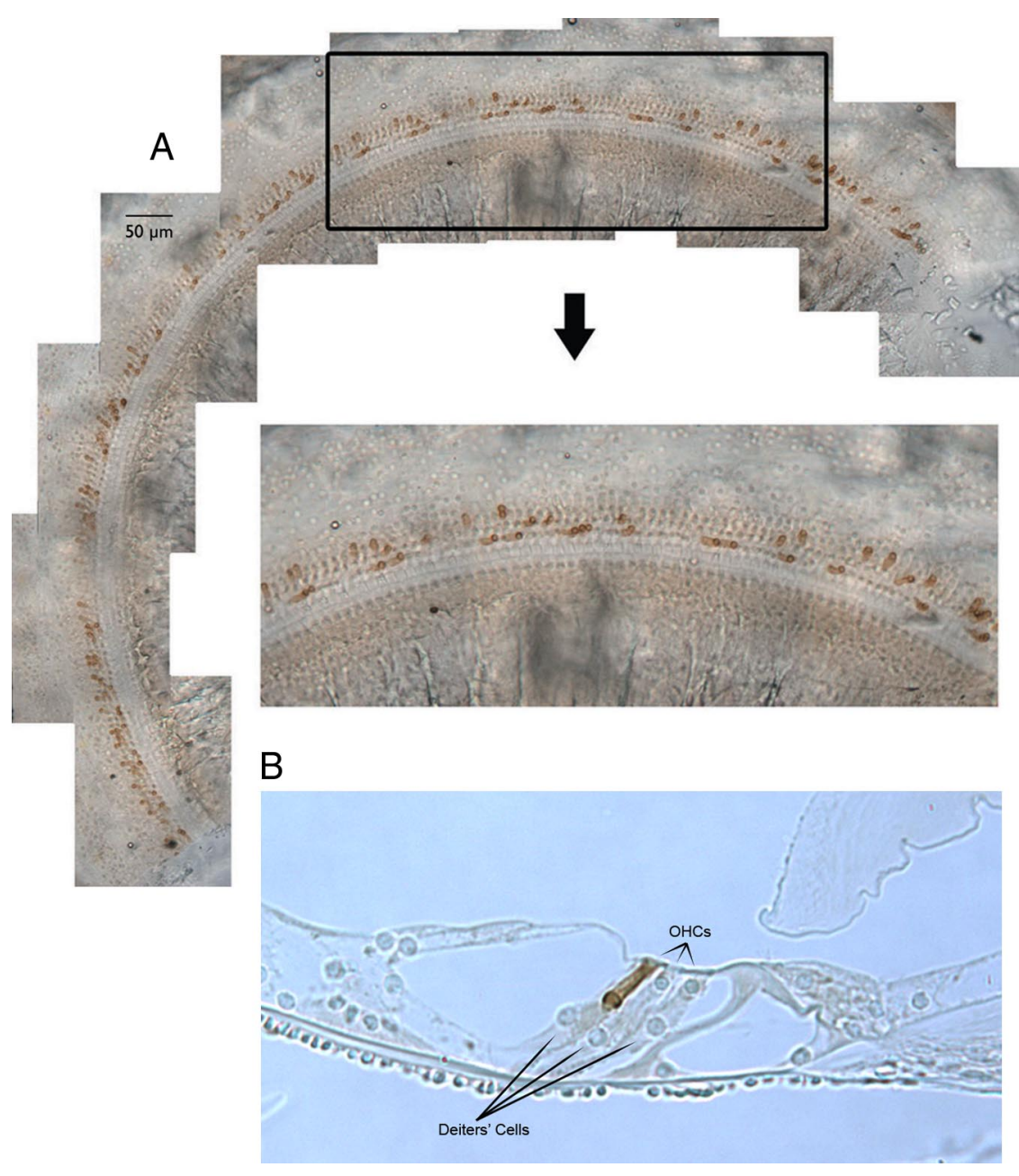

Figure 4. A, Distribution of prestin-containing $\mathrm{OHCs}$ in a chimeric cochlea. A segment of stitched segments lying 2.8 and $4.3 \mathrm{~mm}$ from the base is enlarged to reveal the mosaic pattern of OHCs derived from ROSA26 WT and prestin K0 embryos. Only OHCs from the ROSA26-positive parent stain with anti-prestin. $\boldsymbol{B}$, A representative radial section of the organ of Corti in a chimeric mouse is provided in $\boldsymbol{B}$ for illustrative purposes. This $5 \mu \mathrm{m}$ section was obtained from a mid-cochlear segment ( $\sim 3 \mathrm{~mm}$ from the helicotrema) at $20 \times$ to show increase in Deiters'-cell lengths associated with short, no prestin-containing $\mathrm{OHCs}$. This particular chimera had $39 \% \mathrm{OHCs}$ staining for prestin. The two shorter than normal $\mathrm{OHCs}$ were derived from the prestin $\mathrm{KO}$ parent; the longer $\mathrm{OHC}$ in row 3, from the ROSA26 WT parent.

in the organ. As a result, the reticular lamina remains relatively flat in this ear where, on average, $61 \%$ of the OHCs lack prestin. In fact, our examination of radial sections in several chimeric mice did not reveal any obvious distortions in the reticular lamina or in the overall architecture of the organ of Corti. It is possible, however, that the mechanical properties of the organ of Corti changed in response to an increase in Deiters' cell length. Because there is no significant difference in length between $\mathrm{OHCs}$ in different rows (Keiler and Richter, 2001) (our unpublished observations), the changes in length shown in Figure $4 \mathrm{~B}$ cannot arise as a result of the particular row in which a given $\mathrm{OHC}$ finds itself. In addition, the length of the $\mathrm{OHC}$ in row 3 is consistent with that reported by Keiler and Richter (2001), indicating that WT OHCs do not adjust in the same way as Deiters' cells. In fact, the length of the WT cell in Figure $4 B$ is nearly identical to the lengths of $\mathrm{OHCs}$ in a nearby radial section where all OHCs were derived from the WT parent.

By counting the number of OHCs with and without prestin, cochleograms were constructed as shown in Figure 5. In this figure, the percentage of OHCs lacking prestin is plotted versus percentage distance from the apex; i.e., the base of the cochlea is on the right. Data are included for two brown mice (dashed lines) that have virtually no unstained OHCs. In other words, all OHCs originated from the ROSA26 embryo and all contained prestin. Of the 31 black mice, none had stained cells. The data for black and brown mice indicate that tissue chimerism was not detected in any mouse having a single coat color. Mottled mice plotted with colored lines display a varied distribution of prestin-containing cells, which spans the range from all $\mathrm{OHCs}$ staining for prestin to all OHCs lacking prestin. The cochleogram plotted with dotted pink line indicates that tissue is missing in the apex. The isolated open circle provides the only apical data for this animal. Although absolute counts are not provided, they can be estimated since each $7 \%$ segment contained $170 \pm 8$ OHCs; i.e., the average cochlea contained $2431 \pm 45$ OHCs. Because prestin $\mathrm{KO}$ mice suffer $\mathrm{OHC}$ loss beginning at the base of the cochlea after P21 (Wu et al., 2004), we documented the number of missing $\mathrm{OHCs}$ in chimeric mice. In fact, the percentage of $\mathrm{OHCs}$ lacking prestin in Figure 5 is composed of both unstained and missing cells since it is assumed that missing cells in the base very likely originated from the KO parent. This assumption is based on the observation that no missing cells were found in mice whose organs of Corti contained at least $40 \%$ prestin. Only when mice became more KO-like, did they show an increasing number of missing OHCs at the base of the cochlea. In other words, the chimeric approach is no cure for the OHC death problem in cells lacking prestin. We also evaluated the asymmetry between right and left ears in mottled mice as shown in Figure 6. The percent difference between cochleograms for right and left ears is plotted versus distance. Individual curves appear as dotted lines; mean and SD as solid lines. On average, right and left ears differ by $\sim 18 \%$. For comparison, asymmetry in brown mice was found to be $<2 \%$.

To evaluate the effect of reducing the number of prestincontaining cells, physiological data were obtained using a roundwindow electrode in anesthetized mice. In Figure $7 A$, we show mean data from WT and KO mice plotted with SDs. Brown mice (dashed lines) have WT-like sensitivity and black mice (dotted lines) are similar to KOs in that they have an $\sim 50 \mathrm{~dB}$ threshold shift. These data indicate that a small number of either WT or mutant cells does not dominate the phenotype. Figure $7 B$ provides mean WT and mean prestin KO CAP thresholds (solid black lines) along with results obtained in mottled mice and plotted as colored dashed lines. The range of change in sensitivity spans that encompassed by the two parental strains from near normal to near KO-like, such that sensitivity in mottled mice improved concomitant with an increase in the proportion of WT OHCs. Mottled mice with the worst sensitivity were found to contain $<5 \%$ OHCs with prestin. Because these animals had a slightly 


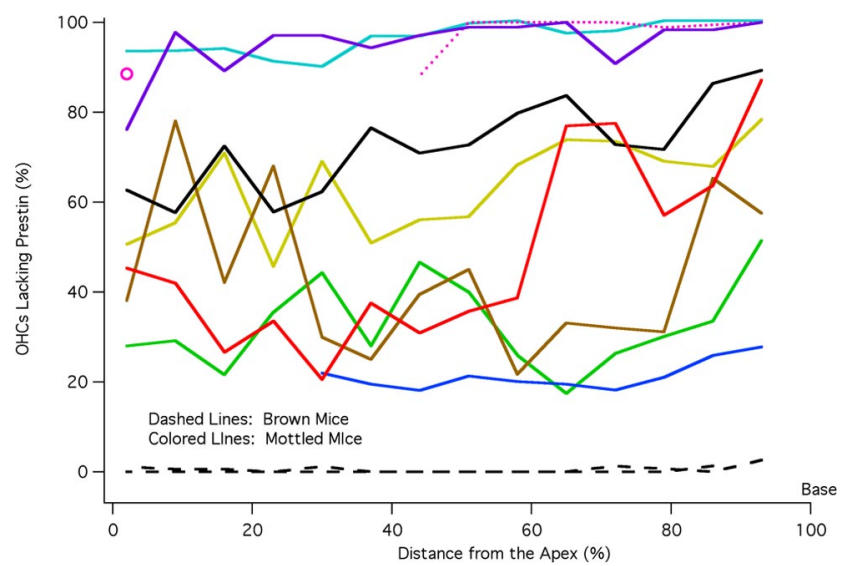

Figure 5. Cochleograms. The percentage of $\mathrm{OHCs} \mathrm{lacking} \mathrm{prestin} \mathrm{is} \mathrm{plotted} \mathrm{as} \mathrm{a} \mathrm{function} \mathrm{of}$ distance from the apex of the cochlea. The average number of unstained/missing cells is computed for each $7 \%$ segment. Cochleograms for brown mice are plotted with dashed lines. Data for mottled mice are represented with solid, colored lines except for the one mouse plotted with pink dotted lines to indicate that data are missing in the apex. Although the data plotted represent the average percentage of $\mathrm{OHCs} \mathrm{lacking} \mathrm{prestin} \mathrm{for} \mathrm{all} \mathrm{rows,} \mathrm{it} \mathrm{should} \mathrm{be} \mathrm{stated} \mathrm{that}$ there was only one instance where a statistically significant difference existed between rows of OHCs. This occurred in one mottled mouse and for only the most basal $7 \%$ section. The color code used for mottled mice is maintained in Figures $7 B, 8$, and 9 .

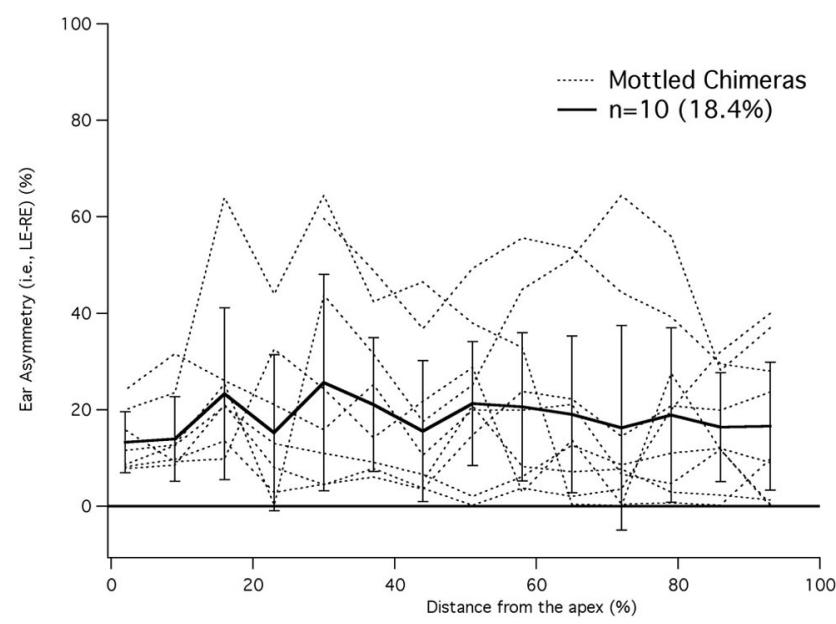

Figure 6. Asymmetry in chimeric ears. The absolute difference between right and left ears is plotted in percent for chimeric mice. The mean difference and SD are also appended and plotted with solid lines.

mottled coat color, we inspected the untested ear and found a larger complement of prestin-staining cells; hence, their mottled appearance. The only condition where the physiological data were inconsistent with coat color was when the ears were asymmetrical and we recorded from the ear with the smallest number of prestin-containing OHCs.

Tuning curves for the CAP were also recorded for a $12 \mathrm{kHz}$ probe tone, as shown in Figure $8 \mathrm{~A}$. Brown mice (solid lines) have normal frequency selectivity and normal tip-to-tail ratios, while the black mice (dotted lines) lack tuning. In contrast, the mottled mice plotted as colored dashed lines show near-normal tuning when probe levels are low; i.e., when they have WT-like sensitivity. However, as mice lose prestin and probe level increases, the tips of the curves move up and the tip-to-tail ratio decreases. For reference, data are appended as solid circles to show a WT tuning curve obtained for a $90 \mathrm{~dB}$ probe. This level is comparable to that used for prestin-KO mice (Cheatham et al., 2007). Although the
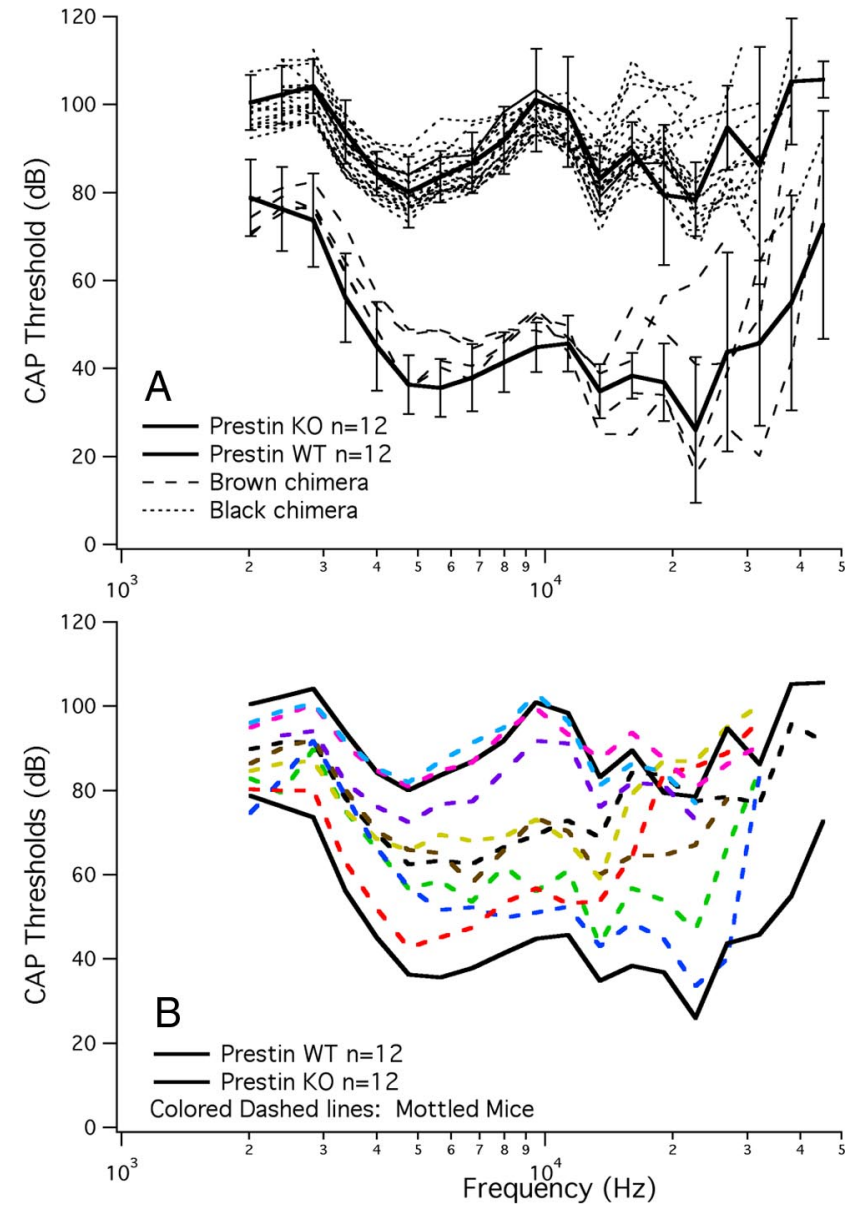

Figure 7. CAP thresholds for chimeric mice. A, Mice of a single coat color appeared as WT when brown (dashed lines) or $\mathrm{KO}$ (dotted lines) when black. For comparison, mean and SDs for WT and prestin KO mice are appended. CAP thresholds in mottled mice are provided in $\boldsymbol{B}$. Animals with a variegated coat color (colored dashed lines) exhibited an intermediate phenotype that spanned the range between WT and KO sensitivities. The more brown in coat color the more WT in phenotype.

tip-to-tail ratio is moderately reduced, WT mice continue to exhibit tuning even at high-probe levels (Dallos and Cheatham, 1976). In other words, loss of tuning in black mice is predominantly due to the absence of prestin-expressing cells and not simply due to increasing probe level.

The relationship between tuning-curve shape and prestin expression is represented in Figure $8 B$ where tip-to-tail ratio is plotted as a function of the average percentage of OHCs lacking prestin in the region lying $30 \%$ to $44 \%$ of the distance from the apex. Based on the mouse frequency-place map (Müller et al., 2005 ), the $12 \mathrm{kHz}$ location is $\sim 33 \%$ from the apex, i.e., in the middle of the $30 \%$ segment, which is $30-37 \%$ of the distance from the apex. Because of the relatively high probe levels used for mottled mice, the average percentage of OHCs without prestin was computed for an extended region in the basal direction to account for the possibility that a larger number of single auditory nerve fibers were responding to the $12 \mathrm{kHz}$ probe; i.e., the average number of $\mathrm{OHCs}$ lacking prestin was computed for two $7 \%$ segments: the one containing the $12 \mathrm{kHz}$ place and the neighboring basal segment. Data from brown mice (open triangle) indicate that they have an average tip-to-tail ratio of $45 \mathrm{~dB}$ and only $1.2 \%$ of OHCs lack prestin. For mottled mice (colored circles), the ratio decreases as the number of OHCs lacking prestin increases. For example, a line fit to the data points indicates that a $50 \%$ 

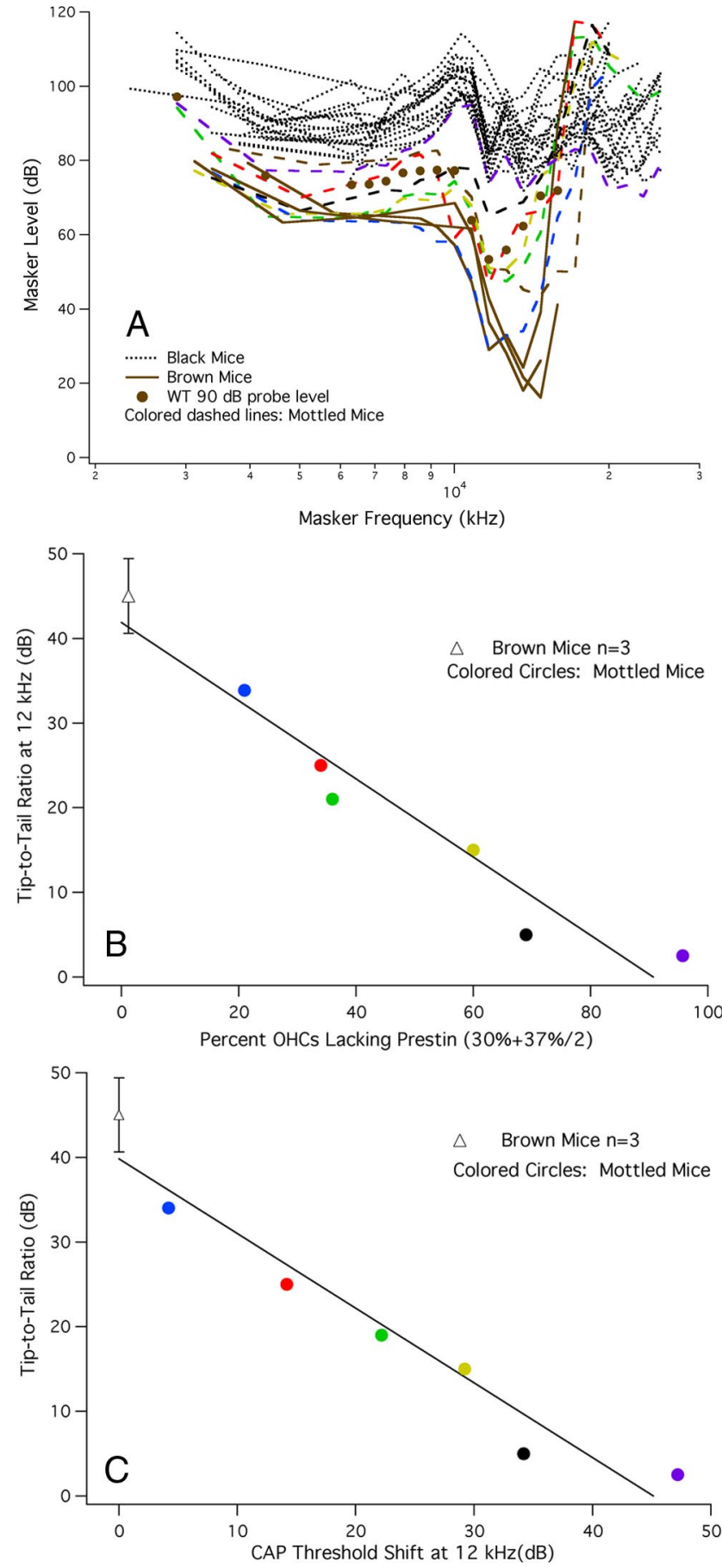

Figure 8. A, CAP tuning curves at $12 \mathrm{kHz}$ in chimeric mice. Similar to the changes in threshold, CAP tuning curves at $12 \mathrm{kHz}$ were WT like in brown mice (solid lines) and KO like in black mice (dotted lines). Mottled mice (colored dashed lines) exhibited a decreasing tip-to-tail ratio as the number of $\mathrm{OHCs}$ containing prestin decreased. The average probe level for brown mice was $54 \mathrm{~dB}$, for black mice, $93 \mathrm{~dB}$ and for mottled mice $74 \mathrm{~dB}$. B, For brown and mottled mice, the tip-to-tail ratio is plotted as a function of the percentage of $\mathrm{OHCs}$ lacking prestin. This latter metric is computed over a distance located between 30 and $44 \%$ of the distance from the apex. Brown mice (open triangle) are represented by their mean and SD, while data for mottled, chimeric mice are plotted individually as colored circles. $C$, In this panel, tip-to-tail ratio is plotted as a function of CAP threshold shift at $12 \mathrm{kHz}$ for brown mice, represented by their mean and SD, and for mottled mice plotted individually. It should be understood that a full dataset was not collected on each mouse. This related to shipping difficulties such that some mice arrived when they were much older than is ideal for making round-window recordings. In addition, some mice displayed anatomical anomalies that prevented CAP recording and in other instances tissue was lost in the region coding for the $12 \mathrm{kHz}$ probe. Hence, our inability to provide results for a larger number of mottled mice.
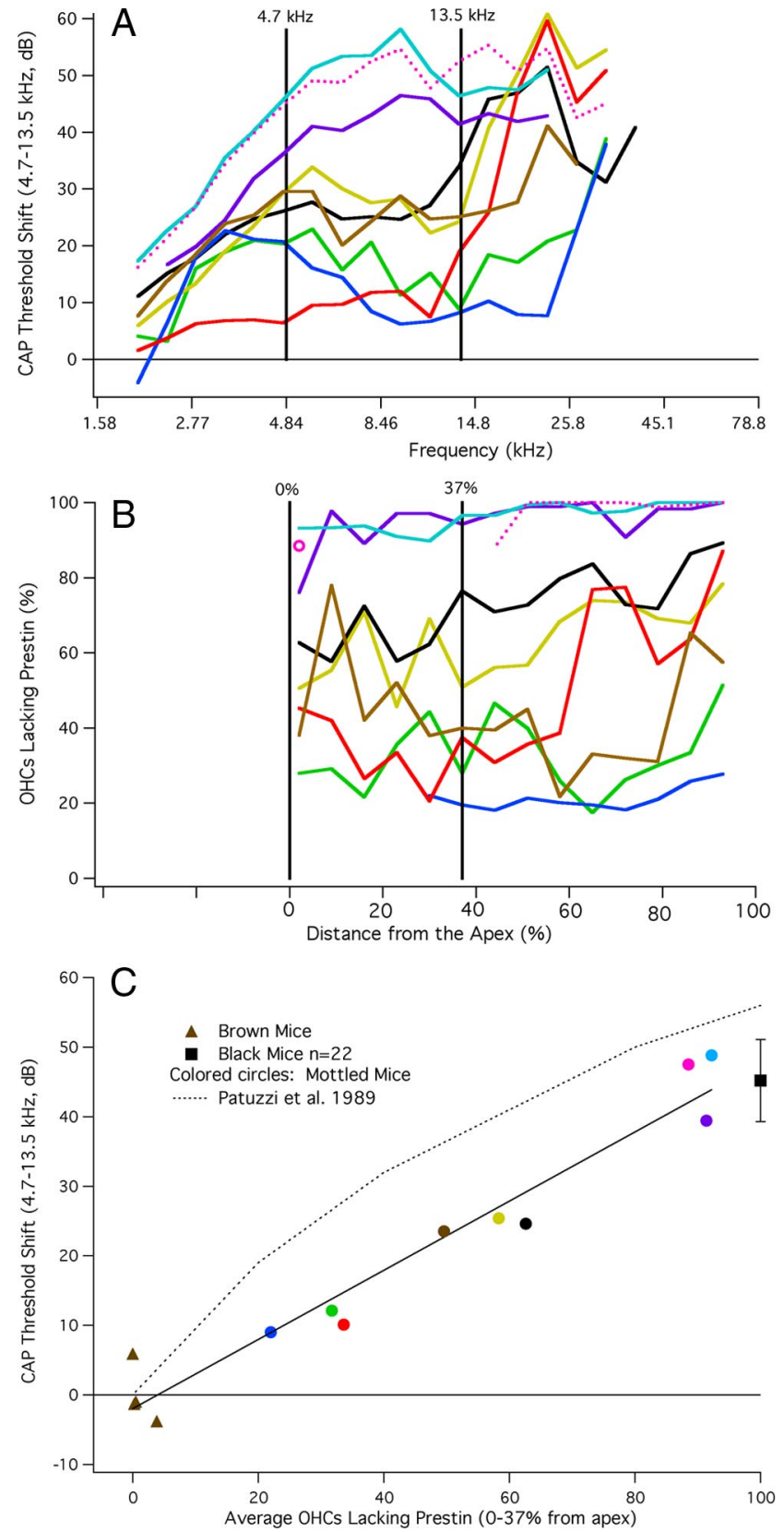

Figure 9. $\quad A, B, C A P$ threshold shifts $(\boldsymbol{A})$ and cochleograms $(\boldsymbol{B})$. By using the mouse frequency/place map of Müller et al. (2005), the physiological and anatomical data were plotted on the same abscissa to correlate changes in prestin expression with changes in sensitivity. It should be noted, however, that CAP responses below $\sim 4.5 \mathrm{kHz}$ are produced by single units with characteristic frequencies greater than the stimulus frequency. In other words, these nerve fibers are responding on the tails of their tuning curves. Average threshold shift and average percentage of $\mathrm{OHCs}$ lacking prestin are computed for frequencies and distances indicated by the vertical lines. The cochleogram plotted with the pink dotted line indicates missing tissue due to dissection artifact. In this case, basal counts are available, as well as for the last apical segment, which appears as an isolated open circle. The CAP threshold shift for this mouse is also plotted with a dotted line. The relationship between $\mathrm{CAP}$ threshold shift and percentage $\mathrm{OHCs}$ lacking prestin is provided in C. In this figure, the average CAP threshold shift for frequencies between 4.7 and $13.5 \mathrm{kHz}$ is plotted as a function of the percentage of $\mathrm{OHCs} \mathrm{lacking} \mathrm{prestin.} \mathrm{It} \mathrm{should} \mathrm{also} \mathrm{be}$ stated that the same result was obtained when the average CAP threshold was extended to 16.5 $\mathrm{kHz}$. However, we decided to use the narrower range because CAP threshold shifts increase rapidly $>13.5 \mathrm{kHz}$ in some mottled mice; i.e., the threshold shift did not remain flat. Data for brown mice appear as triangles, those for mottled mice as colored circles. The mean ( \pm one SD) for black mice appears as a square. The predicted change of gain estimated by Patuzzi et al. (1989, their Fig. 3) is appended for reference. 
reduction in prestin expression is associated with an $\sim 15 \mathrm{~dB}$ tip-tail ratio. The regression line also produces a $0 \mathrm{~dB}$ tip-to-tail ratio at $\sim 75 \%$ reduction in prestin-expressing OHCs. Tip-to-tail ratio is also plotted as a function of CAP threshold shift at $12 \mathrm{kHz}$ in Figure $8 C$. Again, tip length decreases as the sensitivity at probe frequency is reduced due to the loss of prestin-containing OHCs.

In Figure 9, we plot CAP threshold shifts in $A$ and cochleograms showing the percentage of $\mathrm{OHCs}$ lacking prestin in $B$. The frequency/place map of Müller et al. (2005) was again used to determine the anatomical position of each stimulus frequency used to obtain CAP thresholds. Because the two datasets are now plotted on the same abscissa, one can better compare the change in sensitivity with the number of $\mathrm{OHCs}$ lacking prestin. To quantify this relationship, we computed the average threshold shift between 4.7 and $13.5 \mathrm{kHz}$ and correlated this metric with the average number of $\mathrm{OHCs}$ lacking prestin at the locations associated with these frequencies. The frequency range was not extended to higher frequencies for two reasons. First, because variability in measured sensitivity at high frequencies can relate to several causes (anesthesia, temperature, age-related hearing loss, vulnerability) independent of prestin expression and second, because the threshold shifts and the percentage of OHCs lacking prestin are relatively flat in the apical part of the cochlea. Calculations for the region indicated by the vertical lines are shown in Figure 9C. Data points represent the average threshold shift plotted against the average percentage of OHCs lacking prestin for each individual mouse. As expected, brown mice (triangles) have very few cells without prestin and no threshold shift. In other words, they are WT like. Black mice (isolated square \pm one SD) have a large threshold shift and all OHCs lack prestin. The colored circles represent chimeric mice with varying degrees of prestin staining and varying degrees of threshold shift. These results are best fit with a straight line. Also appended are Patuzzi et al.'s (1989, their Fig. 3, dotted line) predictions of gain change (CAP threshold shift) associated with decrease in the cochlear microphonic produced by acoustic trauma. Their empirical estimate of this relationship implies that a $50 \%$ decrease in prestin should produce a $36 \mathrm{~dB}$ threshold shift. Model results of Neely and Kim (1986) indicate an even larger threshold shift, i.e., $56 \mathrm{~dB}$ when the negative damping component of their active hydrodynamic model is reduced by $50 \%$. All data from mottled chimeras are well below these predictions.

\section{Discussion}

Since their introduction in the 1960s, chimeric mice have been shown to provide a valuable tool for testing gene function (LowZeddies and Takahashi, 2005). In this report, anatomical data reveal that the distribution of prestin-containing cells forms a mosaic, consistent with previous chimeric analyses of other genes (Dewey et al., 1976; Oster-Granite and Gearhart, 1981; Goldowitz, 1987; Low-Zeddies and Takahashi, 2001; Guo et al., 2004). In fact, there are studies showing a mosaic expression in the inner ear for frizzled 3 and frizzled 6 (Wang et al., 2006), as well as for math 1 chimeric mice (Du et al., 2007). These recent anatomical results demonstrate a mixture of normal and mutant cells in both cochlear and vestibular sense organs. The observed intermingling of cells derived from the two parental strains implies that a single progenitor cell does not give rise to a large number of OHCs. In other words, OHCs do not seem to descend from a small number of progenitor cells as in the cerebellum (Vogel and Herrup, 1993). In contrast, the cochlea appears to be composed of thousands of $\mathrm{OHCs}$, each derived from a single or very small number of precursor cells, similar to the retina (Williams and Goldowitz, 1992).

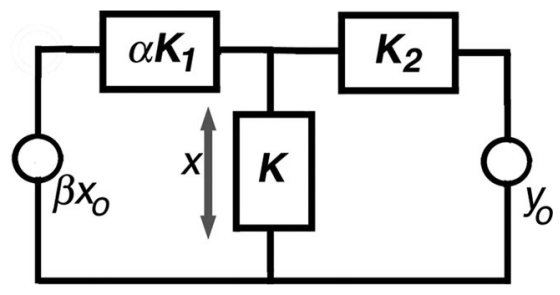

Figure 10. A Thèvenin equivalent circuit showing displacement sources for both somatic $\left(x_{0}\right)$ and ciliary $\left(y_{0}\right)$ motility. Component factors are identified in the Discussion.

This is consistent with the observation that OHCs exhibit a primarily vertical nuclear migration (Katayama and Corwin, 1993) from their place of generation, to their adult position within the organ of Corti.

We also found no tissue chimerism in mice with a single coat color, similar to the clock chimera (Low-Zeddies and Takahashi, 2001). For example, brown mice have $>95 \%$ OHCs with prestin, while black mice showed no prestin staining. Although we found asymmetry between ears, the average asymmetry was $\sim 18 \%$. This result compares well with observations in the nervous system where the reported differences in cell proportions between sides was usually $<25 \%$ (Sanyal and Zeilmaker, 1977; Herrup et al., 1984a,b; Herrup, 1986; Herrup and Sunter, 1986; Crandall and Herrup, 1990; Fishell et al., 1990). Except in mice with asymmetrical ears, there was a correlation between coat color and the anatomical/physiological measurements in the cochlea. It should also be emphasized that we did not observe a mixed physiological phenotype in which the proportion of prestin-containing cells impacted sensitivity independent of tuning. In other words, if there was a change in threshold, there was a decrease in tip-to-tail ratio, i.e., a decrease in tuning curve tip length. Hence, these observations are consistent with the notion that OHCs containing prestin are essential for the sensitivity and frequency selectivity expressed in the organ of Corti and that the mutant defect is intrinsic to the $\mathrm{OHC}$; i.e., the prestin effect acts in a cellautonomous mode.

To validate this approach, we made additional controls by mating a ROSA26 WT male with a prestin KO female. All offspring showed lacZ staining and normal cochlear function. The latter result is consistent with Cheatham et al. (2005) where nearnormal levels of prestin protein were produced in prestin heterozygotes, thereby preserving WT-like sensitivity and frequency selectivity. It was also demonstrated that cells in mice having only one copy of the prestin gene were similar to WT OHCs in length and although not measured, probably in stiffness as well.

When making comparisons between this control and a chimera, it should be emphasized that all OHCs in mice containing only one copy of WT prestin are heterozygous for the prestin gene. In contrast, OHCs in the chimera are either WT or KO, i.e., no $\mathrm{OHC}$ is heterozygous for the prestin gene. In a 50:50 balanced chimera, half of the OHCs express prestin and half do not; i.e., half of the cells are short and much less stiff than their neighbors. In mice with only one copy of the prestin gene, the OHCs are all the same; i.e., they are near normal in protein, length and most likely stiffness. So the observation that ROSA26 WT/prestin $\mathrm{KO}$ controls have normal hearing simply indicates that changes in genetic background and the presence of the reporter gene lac $Z$ do not impact phenotype. This result, however, does not contradict the observation that a 50:50 chimera shows about half of the maximum gain.

In contrast to $\mathrm{KO} / \mathrm{KI}$ mouse models, an intermediate phenotype is observed in prestin chimeric mice such that a partial loss of 
gain is recorded. In other words, the CAP thresholds and CAP tuning curves in mottled mice exhibit a phenotypic gradient, where the transition between WT animals having normal amplification and KOs having none occurs in proportion to their constituent genotypes. Hence, the data indicate a proportional change in amplifier gain (in decibels) as the number of prestincontaining $\mathrm{OHCs}$ decreases; i.e., genotype ratio and phenotype are intimately related in prestin chimeric mice. The loss of sensitivity, however, is smaller than predictions from current models of amplification. There is also no evidence of nonlinearity in the relationship between sensitivity and amplifier gain, which characterizes Patuzzi et al.'s (1989) model predictions, as well as those of Neely and Kim (1986).

One can approach these results in a semiquantitative way. In Figure 10, a Thèvenin equivalent is shown for a length of cochlea within which the outputs of OHCs sum. We assume that there are two separate displacement sources: OHC somatic motility $\left(\beta x_{\mathrm{o}}\right)$ and stereociliary motility $\left(y_{\mathrm{o}}\right)$. The factor $\beta$ represents the fraction of $\mathrm{OHCs}$ producing somatic motility, i.e., those containing prestin. The source compliance of the somatic motor complex is $\alpha K_{1}$, while that of the ciliary motor complex is $K_{2}$. The factor $\alpha$ represents change in compliance due to prestin removal. The load compliance, $K$, symbolizes the passive mechanical load upon the motors, principally some combination of reticular lamina, tectorial membrane and basilar membrane compliances. Neither absolute nor relative magnitudes matter a great deal in the simple exercise that follows.

Let us express the displacement across the load as $x$. If only $x_{\mathrm{o}}$ is active, then

$$
x=x_{\mathrm{o}} \frac{\beta K K_{2}}{K K_{2}+\alpha K_{1}\left(K+K_{2}\right)} .
$$

Next, we compute $x$ when only $y_{\mathrm{o}}$ is active, such that

$$
x=y_{\mathrm{o}} \frac{\alpha K_{1} K}{\alpha K_{1} K+K_{2}\left(\alpha K_{1}+K\right)} .
$$

Notice that as $\beta$ decreases, so does the displacement due to somatic motility, but there is no change in ciliary-driven displacement. Next, the compliance of somatic OHC motors is varied between a normal, wild-type prestin value of $\alpha=1$ and an extreme value of infinite compliance. In this second case, the displacement, $x$, decreases when the driving source is $x_{0}$, but the response increases for the ciliary drive, $\mathrm{y}_{\mathrm{o}}$, because the denominator decreases in Equation 2. Assuming that feedback/gain is provided by somatic motility, a decrease in the number of contributing OHCs or an increase in source compliance yields a reduced response. This circuit analogy suggests that the reduction of prestin/stiffness in chimeric mice serves to decouple the system, thereby diminishing the interaction of OHCs with other elements in the feedback loop. This effect would very likely preclude any amplifier from generating enough force to boost basilar membrane vibrations at lowstimulus levels. Because the ciliary amplifier is demonstrated to increase mechanical displacements when compliance increases, this simulation, as well as the chimera data, do not agree with a model in which the principal source of gain is ciliary motility alone.

A chimera analysis of prestin function indicates proportionality between threshold shift and amplification, as measured by CAP thresholds and the tip-length of CAP tuning curves, and between these variables and the proportion of prestin-expressing
$\mathrm{OHCs}$ in the cochlea. The change in threshold versus proportion of no-prestin OHCs in the amount of $\sim 0.5 \mathrm{~dB} / \%$ is a useful estimate of the logarithmic relationship between these variables. These results are consistent with prestin-mediated OHC motility playing a critical role in cochlear amplification.

\section{References}

Bohne BA, Harding GW (1997) Processing and analyzing the mouse temporal bone to identify gross, cellular and subcellular pathology. Hear Res 109:34-45.

Brown SD, Hardisty-Hughes RE, Mburu P (2008) Quiet as a mouse: dissecting the molecular and genetic basis of hearing. Nat Rev Genet 9:277-290.

Cheatham MA, Huynh KH, Gao J, Zuo J, Dallos P (2004) Cochlear function in Prestin knockout mice. J Physiol 560:821-830.

Cheatham MA, Zheng J, Huynh KH, Du GG, Gao J, Zuo J, Navarrete E, Dallos $\mathrm{P}$ (2005) Cochlear function in mice with only one copy of the Prestin gene. J Physiol 569:229-241.

Cheatham MA, Zheng J, Huynh KH, Du GG, Edge RM, Anderson CT, Zuo J, Ryan AF, Dallos P (2007) Evaluation of an independent prestin mouse model derived from the 129S1 strain. Audiol Neurootol 12:378-390.

Crandall JE, Herrup K (1990) Patterns of cell lineage in the cerebral cortex reveal evidence for developmental boundaries. Exp Neurol 109:131-139.

Dallos P, Cheatham MA (1976) Compound action potential (AP) tuning curves. J Acoust Soc Am 59:591-597.

Dallos P, Harris D (1978) Properties of auditory nerve responses in the absence of outer hair cells. J Neurophysiol 41:365-383.

Dallos P, Wu X, Cheatham MA, Gao J, Zheng J, Anderson CT, Jia S, Wang X, Cheng WH, Sengupta S, He DZ, Zuo J (2008) Prestin-based outer hair cell motility is necessary for mammalian cochlear amplification. Neuron 58:333-339.

Dewey MJ, Gervais AG, Mintz B (1976) Brain and ganglion development from two genotypic classes of cells in allophenic mice. Dev Biol 50:68-81.

Du X, Jensen P, Goldowitz D, Hamre KM (2007) Wild-type cells rescue genotypically Math1-null hair cells in the inner ears of chimeric mice. Dev Biol 305:430-438.

Falconer DS, Avery PJ (1978) Variability of chimeras and mosaics. J Embryol Exp Morph 43:195-219.

Fishell G, Rossant J, van der Kooy D (1990) Neuronal lineages in chimeric mouse forebrain are segregated between compartments and in the rostrocaudal and radial planes. Dev Biol 141:70-83.

Friedrich G, Soriano P (1991) Promotor traps in embryonic stem cells: A genetic screen to identify and mutate developmental genes in mice. Genes Dev 5:1513-1523.

Friedrich VL Jr, Holstein GR, Li X, Gow A, Kelley KA, Lazzarini RA (1993) Intracellular distribution of transgenic bacterial $\beta$-galactosidase in central nervous system neurons and neuroglia. J Neurosci Res 36:88-98.

Gao J, Wang X, Wu X, Aguinaga S, Huynh K, Jia S, Matsuda K, Patel M, Zheng J, Cheatham M, He DZ, Dallos P, Zuo J (2007) Prestin-based outer hair cell electromotility in knockin mice does not appear to adjust the operating point of a cilia-based amplifier. Proc Natl Acad Sci USA 104:12542-12547.

Goldowitz D (1987) Cell partitioning and mixing in the formation of the CNS: Analysis of the cortical somatosensory barrels in chimeric mice. Dev Br Res 35:1-9.

Guo N, Hawkins C, Nathans J (2004) Frizzled 6 controls hair patterning in mice. Proc Natl Acad Sci U S A 101:9277-9281.

Herrup K (1986) Cell lineage relationships in the development of the mammalian CNS: role of cell lineage in control of cerebellar Purkinje cell number. Dev Biol 115:148-154.

Herrup K, Sunter K (1986) Cell lineage dependent and independent control of Purkinje cell number in the mammalian CNS: Further quantitative studies of lurcher chimeric mice. Dev Biol 117:417-427.

Herrup K, Diglio TJ, Letsou A (1984a) Cell lineage relationships in the development of the mammalian CNS. I. The facial nerve nucleus. Dev Biol 103:329-336.

Herrup K, Wetts R, Diglio TJ (1984b) Cell lineage relationships in the development of the mammalian CNS. II. Bilateral independence of CNS clones. J Neurogenet 1:275-288.

Hogan B, Beddington R, Costantini F, Lacy E (1994) Manipulating the mouse embryo. New York: Cold Spring Harbor.

Katayama A, Corwin JT (1993) Cochlear cytogenesis visualized through pulse labeling of chick embryos in culture. J Comp Neurol 333:28-40. 
Keiler S, Richter CP (2001) Cochlear dimensions obtained in hemicochleae of four different strains of mice: CBA/CaJ, 129/CD1, 129/SvEv and C57BL/6J. Hear Res 162:91-104.

Kelly SJ (1977) Studies of the developmental potential of 4- and 8-cell stage mouse blastomeres. J Exp Zool 200:365-376.

Liberman MC, Gao J, He DZ, Wu X, Jia S, Zuo J (2002) Prestin is required for electromotility of the outer hair cell and for the cochlear amplifier. Nature 419:300-304.

Low-Zeddies SS, Takahashi JS (2001) Chimera analysis of the Clock mutation in mice shows that complex cellular integration determines circadian behavior. Cell 105:25-42.

Low-Zeddies SS, Takahashi JS (2005) Mouse chimeras and their application to circadian biology. Meth Enzymol 393:478-492.

Matsuda K, Zheng J, Du GG, Klöcker N, Madison LD, Dallos P (2004) $\mathrm{N}$-linked glycosylation sites of the motor protein prestin: effects on membrane targeting and electrophysiological function. J Neurochem 89:928-938.

Mintz B (1962) Formation of genotypically mosaic mouse embryos. Am J Zoo 4:432.

Mullen RJ, Whitten RK (1971) Relationship of genotype and degree of chimerism in coat color to sex ratios and gametogenesis in chimeric mice. J Exp Zoo 178:165-176.

Müller M, von Hünerbein K, Hoidis S, Smolders JW (2005) A physiological place-frequency map of the cochlea in the CBA/J mouse. Hear Res 202:63-73.

Neely ST, Kim DO (1986) A model for active elements in cochlear mechanics. J Acoust Soc Am 79:1472-1480.

Oster-Granite ML, Gearhart J (1981) Cell lineage analysis of cerebellar Purkinje cells in mouse chimeras. Dev Biol 85:199-208.

Patuzzi RB, Yates GK, Johnstone BM (1989) Changes in cochlear micro- phonic and neural sensitivity produced by acoustic trauma. Hear Res 39:189-202.

Ryan A, Dallos P (1975) Effect of absence of cochlear outer hair cells on behavioural auditory threshold. Nature 253:44-46.

Sanyal S, Zeilmaker GH (1977) Cell lineage in retinal development of mice studied in experimental chimaeras. Nature 265:731-733.

Tarkowski AK (1961) Mouse chimaeras developed from fused eggs. Nature 190:857-860.

Vogel MW, Herrup K (1993) A theoretical and experimental examination of cell lineage relationships among cerebellar Purkinje cells. Dev Biol 156:49-68.

Wang Y, Guo N, Nathans J (2006) The role of Frizzled3 and Frizzled6 in neural tube closure and in the planar polarity of inner-ear sensory hair cells. J Neurosci 26:2147-2156.

Williams RW, Goldowitz D (1992) Structure of clonal and polyclonal cell arrays in chimeric mouse retina. Proc Natl Acad Sci U S A 89:1184-1188.

Wu X, Gao J, Guo Y, Zuo J (2004) Hearing threshold elevation precedes hair-cell loss in prestin knockout mice. Brain Res Mol Brain Res 126:30-37.

Zambrowicz BP, Imamoto A, Fiering S, Herzenberg LA, Kerr WG, Soriano P (1997) Disruption of overlapping transcripts in the ROSA $\beta$ geo 26 gene trap strain leads to widespread expression of $\beta$-galactosidase in mouse embryos and hematopoietic cells. Proc Natl Acad Sci U S A 94:3789-3794.

Zheng J, Shen W, He DZ, Long KB, Madison LD, Dallos P (2000) Prestin is the motor protein of cochlear outer hair cells. Nature 405:149-155.

Zheng J, Long KB, Matsuda KB, Madison LD, Ryan AD, Dallos PD (2003) Genomic characterization and expression of mouse prestin, the motor protein of outer hair cells. Mamm Genome 14:87-96. 